\title{
Automatic Distance Measurement and Material Characterization with Infrared Sensors*
}

\author{
Miguel Angel Garcia and Agusti Solanas \\ Intelligent Robotics and Computer Vision Group, \\ Department of Computer Science and Mathematics, \\ Rovira i Virgili University, \\ Av. Països Catalans 26, 43007 Tarragona, Spain \\ \{magarcia, asolanas\} @etse.urv.es
}

\begin{abstract}
This paper describes a new technique for determining the distance to a planar surface and, at the same time, obtaining a characterization of the surface's material through the use of conventional, low-cost infrared sensors. The proposed technique is advantageous over previous schemes in that it does not require additional range sensors, such as ultrasound devices, nor a priori knowledge about the materials that can be encountered. Experiments with an all-terrain mobile robot equipped with a ring of infrared sensors are presented.
\end{abstract}

\section{Introduction}

Infrared sensors are commonly utilized in mobile robotics as low-cost proximity sensors, basically for immediate collision avoidance. Their non-linear behavior and high dependence on the reflectivity of the sensed objects has prevented their application as range sensors, in favor of more sophisticated and costly devices, such as sonar (ultrasound) or laser systems.

Notwithstanding, since infrared sensors are inexpensive and readily available in most commercial mobile robots, some researchers have tried to overcome their limitations. A pioneering work is due to Novotny and Ferrier [4]. They applied the Phong illumination model [5] in order to compute the distance to a planar surface and, simultaneously, determine two model coefficients that represent the reflective properties of that surface. Unfortunately, this technique also requires a second ring of ultrasound sensors, each conveniently placed next to an infrared sensor, in order to obtain a parameter that is crucial to the solution of the problem: the minimum distance $d$ between the robot and the surface being measured, Fig. 1.

In a similar direction, [2] presents a technique for computing distances to unknown planar surfaces by means of infrared sensors. In this case, a simpler illumination model based on the photometry inverse square law is utilized. This model characterizes the reflectivity of the surface being measured with a single coefficient. Once that coefficient has been established, the infrared sensor readings can be directly mapped to distances. However, that crucial coefficient must be estimated from the distance to the surface, which is also measured by an ultrasound sensor.

\footnotetext{
* This work has been partially supported by the Government of Spain under the CICYT project DPI2001-2094-C03-02.
} 


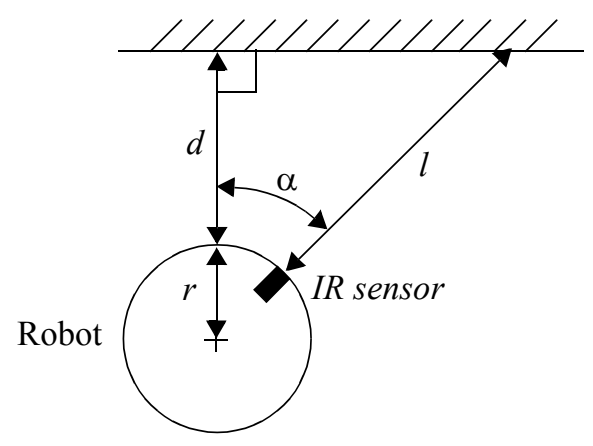

Fig. 1. Diagram of a robot emitting an infrared signal, adapted from [4]

Following a different approach, [1] presents a technique that applies infrared sensors for determining the shape of an unknown surface from among four basic shapes (plane, corner, edge and cylinder) independently of its position. When the shape is known, the distance can also be estimated. No ultrasound sensors are necessary. However, this technique requires an off-line, supervised training stage for generating an exhaustive collection of reference angular scans that describe the sensor output as a function of the incidence angle ( $\alpha$ in Fig. 1) for all types of materials that can be encountered, the four considered shapes, and different distances (at regular increments of $2.5 \mathrm{~cm}$ ). When a real scan is acquired, the system finds a matching reference scan within the database. Such an exhaustive training with a limited number of materials can be a serious limitation that may prevent the application of this technique to real situations in which there is not a priori knowledge of the kind of surfaces that can be encountered, especially in case of exploration tasks.

Based on the theory presented in [4], the present paper describes a new technique that exclusively utilizes low-cost infrared sensors for estimating the reflective coefficients of an unknown planar surface and then computing its distance to a robot, without any prior training or knowledge about the materials that can be encountered in the scene. The technique only requires two angular scans of the unknown surface from two positions from where the surface can be detected, such that the distance between both positions is known. In our experiments, this is done with a single infrared sensor mounted at the front of a robot. The robot rotates around its center to perform the first scan, then moves a predefined distance toward or against the surface and scans it again. The whole process can be performed with a single scan by using two infrared sensors mounted on concentric circles of different known radius.

This paper is organized as follows. The proposed technique is described in Section 2. Experimental results are discussed in Section 3. Finally, conclusions and further improvements are presented in Section 4.

\section{Surface Characterization and Distance Measurement}

The infrared sensors considered in this work are constituted by an infrared LED emitter and a photo-diode that measures the amount of emitted energy that is reflected by the surface of a nearby object. 
Based on the Phong's illumination model and the photometry theory, [4] establishes that the energy absorbed by an infrared sensor mounted on a robot at distance $r$ from its rotation center (Fig. 1) can be approximated by:

$$
E_{\alpha}^{d}=\left(C_{0} \cos (\alpha)+C_{1} \cos (2 \alpha)\right) /\left[\frac{d}{\cos (\alpha)}+r\left(\frac{1}{\cos (\alpha)}-1\right)\right]^{2}
$$

where $d$ is the minimum distance between the sensor and the surface, $\alpha$ is the incidence angle of light with respect to the surface and $C_{0}$ and $C_{1}$ are two coefficients that express the reflectivity properties of the surface being sensed. This energy corresponds to the actual sensor reading.

The proposed technique aims at determining the two reflective coefficients and the minimum distance $d$ by means of two angular scans. Each scan consists of a sequence of $N$ sensor readings obtained while the robot and, hence, the sensor, turn around the robot's rotation center. Since the robot rotates at a known angular speed and the sensor is read at a constant frequency, it is straightforward to determine the angular increment between any pair of consecutive readings. From that increment, the relative angle $\alpha$ between the current sensor orientation and the one in which the sensor is perpendicular to the surface and, hence, receives maximum energy, is computed. Let $\Phi$ be the maximum angle (e.g., $\Phi=\pi / 6$ ). When the distance between the sensor and the surface is the sought minimum distance $d$, angle $\alpha$ is zero.

Let $\left\{E_{\alpha}^{d}\right\}$ and $\left\{E_{\alpha}^{d^{\prime}}\right\}, \alpha \in[0, \Phi]$, be two angular scans of $N$ readings obtained at two unknown minimum distances, $d$ and $d^{\prime}, d<d^{\prime}$, whose separation is known:

$$
\Delta=d^{\prime}-d
$$

According to the previous definition of $\alpha, E_{0}^{d}$ and $E_{0}^{d^{\prime}}$ are the maximum energies detected by the sensor in both scans respectively. From (1), the minimum distance to the surface is expressed as [4]:

$$
d=r(\cos (\alpha)-1)+\cos (\alpha) \sqrt{\left(C_{0} \cos (\alpha)+C_{1} \cos (2 \alpha)\right) / E_{\alpha}^{d}}
$$

By combining (2) and (3):

$$
\Delta=\left(1 / \sqrt{E_{\alpha}^{d^{\prime}}}-1 / \sqrt{E_{\alpha}^{d}}\right) \cos (\alpha) \sqrt{C_{0} \cos (\alpha)+C_{1} \cos (2 \alpha)}
$$

Hence:

$$
C_{0} \cos (\alpha)+C_{1} \cos (2 \alpha)=\Delta^{2} /\left(\cos (\alpha)\left(1 / \sqrt{E_{\alpha}^{d^{\prime}}}-1 / \sqrt{E_{\alpha}^{d}}\right)\right)^{2}
$$

Given an orientation $\alpha$ and its corresponding sensor readings, $E_{\alpha}^{d}$ and $E_{\alpha}^{d^{\prime}}$, the previous expression defines a linear equation in which the two unknowns are the reflective coefficients $C_{0}$ and $C_{1}$. Since $N$ readings are available, each with a different $\alpha$, an over determined system of $N$ linear equations over two unknowns is defined and solved by applying least-squares.

Once the two reflective coefficients are known, the minimum distance to the surface is simply found by applying (3) for $\alpha=0$ :

$$
d=\sqrt{\left(C_{0}+C_{1}\right) / E_{0}^{d}}
$$



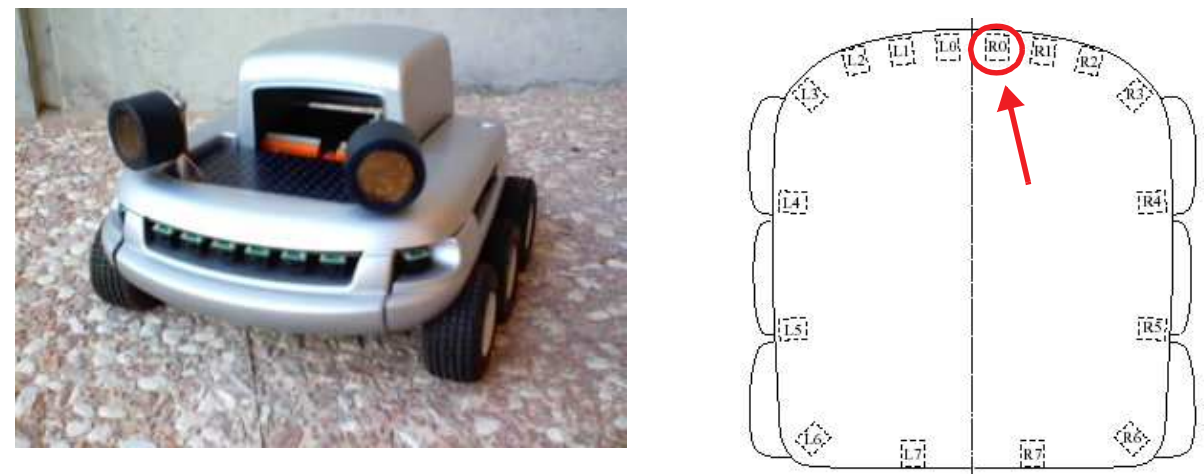

Fig. 2. K-Team's Koala robot equipped with a ring of 16 infrared sensors. The proposed technique is applied to the readings obtained with the highlighted frontal sensor

\section{Experimental Results}

The proposed technique has been tested on real surfaces of different colors and materials by using an all-terrain Koala mobile robot (Fig. 2). This robot is equipped with a ring of 16 infrared sensors distributed around the robot's chassis. In particular, each sensor is a TSL252 consisting of an infrared LED that emits at $880 \mathrm{~nm}$ and a photo-diode that detects the reflected light. Each device generates an output voltage that is read by the robot through a 10-bit A/D converter.

This technique has been applied to the readings obtained by a single frontal sensor highlighted in Fig. 2(right). Once the robot is close to the surface to be measured, it performs a first angular scan by rotating around its center at constant angular speed while the sensor is read at a constant frequency. Once the sequence of readings is complete, the robot turns back to the orientation in which the maximum energy was found - this is the orientation in which $\alpha$ is considered to be zero. At this point the robot moves backwards (against the surface) a predefined distance $\Delta$ and performs a second scan similar to the initial one.

The experiments presented in this paper have been performed with a $\Delta$ equal to 2 $\mathrm{cm}$. Other values are possible and do not significantly affect the results whenever the sensor is kept within the operational range in which it has sufficient accuracy. However, if $\Delta$ is too small, the discretization of the sensor readings will not allow to distinguish variations of light in case the distance to the surface is relatively large.

Fig. 3 shows two examples of angular scans respectively acquired at approximately 10 and $12 \mathrm{~cm}(\Delta=2)$ away from a white thin cardboard, by following the operational procedure described above. The angular resolution between consecutive readings is 0.015 degrees. The maximum energies are $E_{0}^{d}=935$ and $E_{0}^{d^{\prime}}=653$ respectively. These energies are not expressed in physical units but in sensor units (integers ranging between 0 and 1,023).

Therefore, the reflective coefficients, $C_{0}$ and $C_{1}$, do not really have the physical interpretation that the Phong's coefficients have (energy $\times$ distance $^{2}$ ). Instead, they are expressed in terms of sensor units: sensor-units $\times$ distance $^{2}$. This does not pose any problem in order to compute the minimum distance $d$ in the same units as $\Delta(\mathrm{cm}$ 


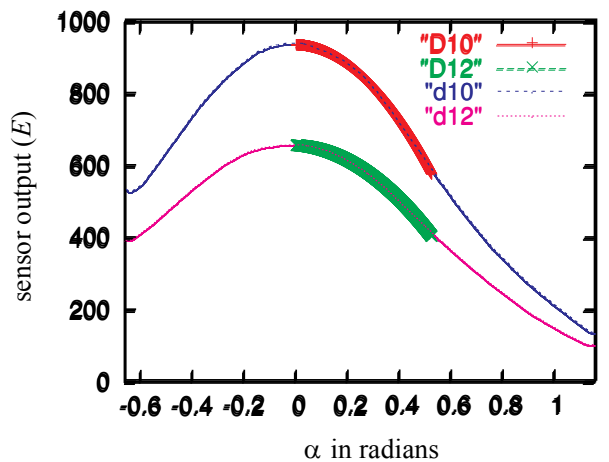

Fig. 3. Two infrared scans acquired at positions separated by $2 \mathrm{~cm}(\Delta=2)$, at minimum distance $d$ approximately equal to $10(\mathrm{~d} 10)$ and $12(\mathrm{~d} 12) \mathrm{cm}$ respectively from a white thin cardboard. The thickened curves (D10 and D12) are sections of the approximating parabolas

in our case), since, according to (6), both $C_{0}$ and $C_{1}$ are divided by $E_{0}^{d}$ and, hence, the sensor units cancel each other.

In order to remove noise inherent to the sensor and compensate for discretization errors due to the $\mathrm{A} / \mathrm{D}$ conversion, the readings $E_{\alpha}^{d}$ and $E_{\alpha}^{d^{\prime}}$ obtained from the sensor are filtered out before applying them to (5). This is done by approximating a secondorder polynomial (a parabola) to the sensed readings comprised between $-\Phi$ and $\Phi$ through least-squares fitting. Within this range of angles, the original curves can be approximated by a second order polynomial without significant error. This filtering stage is also beneficial in order to extrapolate the missing energy readings in case the sensor saturates in being too close to the measured surface. The thickened curves in Fig. 3 correspond to the sections of both parabolas (between 0 and $\Phi$ ) which are utilized in the subsequent estimation process.

An over-determined linear system of $N$ equations (5) is defined and solved by applying a least-squares system equation solver. In the example, the result is $C_{0}=17.7$ and $C_{1}=-8.12$. According to these values and $E_{0}^{d}$ and $E_{0}^{d^{\prime}}$, the minimum distances after applying (6) are: $d=10.14 \mathrm{~cm}$ and $d^{\prime}=12.12 \mathrm{~cm}$.

In order to determine the influence of distance on the computation both of the reflective coefficients, $C_{0}$ and $C_{1}$, and the estimated minimum distance $d$, the same process described above has been carried out at different test distances within the sensor's operational range for various types of material. Each experiment has been run a number of times for every distance. Table 1 and Table 2 show the average results corresponding to white and black thin cardboard respectively. Fig. 4 shows the evolution of the computed distances with respect to the test distances for those two materials. At every test distance, the reflective coefficients have been computed through the procedure described above.

The previous results indicate that the distance estimation starts degrading beyond a certain point away from the measured surface. In our experiments, the distance error is below $1 \mathrm{~mm}$ for distances up to $14 \mathrm{~cm}$ from the surface. From $14 \mathrm{~cm}$ to 18 $\mathrm{cm}$, the error is kept below $1 \mathrm{~cm}$. Beyond this distance, the error progressively degrades. This degradation is inherent to the infrared technology being used and 
Table 1. Average Results for White Thin Cardboard at Different Test Distances

\begin{tabular}{|c||c|c|c|c|c|c|}
\hline \multirow{2}{*}{\begin{tabular}{c}
\multirow{2}{*}{$\begin{array}{c}\text { Test } \\
d \\
(\mathbf{c m})\end{array}$} \\
\cline { 2 - 7 }
\end{tabular}} & \multicolumn{6}{|c|}{ Computed Parameters (average and standard) } \\
\cline { 2 - 7 } & $\bar{d}$ & $\sigma_{d}$ & $\overline{C_{0}}$ & $\sigma_{C_{0}}$ & $\overline{C_{1}}$ & $\sigma_{C_{1}}$ \\
\hline 10 & 10.05 & 0.119 & 16.35 & 1.189 & -7.22 & 0.786 \\
\hline 12 & 11.92 & 0.098 & 16.24 & 0.614 & -7.17 & 0.517 \\
\hline 14 & 13.96 & 0.149 & 15.28 & 0.540 & -6.05 & 0.404 \\
\hline 16 & 15.59 & 0.291 & 13.61 & 0.105 & -5.06 & 0.081 \\
\hline 18 & 17.23 & 0.291 & 13.15 & 0.025 & -4.91 & 0.227 \\
\hline
\end{tabular}

Table 2. Average Results for Black Thin Cardboard at Different Test Distances

\begin{tabular}{|c||c|c|c|c|c|c|}
\hline \multicolumn{1}{|c||}{\multirow{2}{*}{$\begin{array}{c}\text { Test } \\
d \\
(\mathbf{c m})\end{array}$}} & \multicolumn{5}{c|}{ Computed Parameters (average and standard) } \\
\cline { 2 - 7 } & $\bar{d}$ & $\sigma_{d}$ & $\overline{C_{0}}$ & $\sigma_{C_{0}}$ & $\overline{C_{1}}$ & $\sigma_{C_{1}}$ \\
\hline \hline 10 & 10,04 & 0,140 & 13,53 & 0,671 & $-6,30$ & 0,511 \\
\hline 12 & 12,00 & 0,320 & 12,40 & 0,458 & $-5,14$ & 0,172 \\
\hline 14 & 13,87 & 0,497 & 11,53 & 0,270 & $-4,49$ & 0,389 \\
\hline 16 & 15,68 & 0,209 & 10,55 & 0,413 & $-3,72$ & 0,567 \\
\hline 18 & 17,16 & 0,358 & 10,38 & 0,468 & $-3,82$ & 0,277 \\
\hline
\end{tabular}

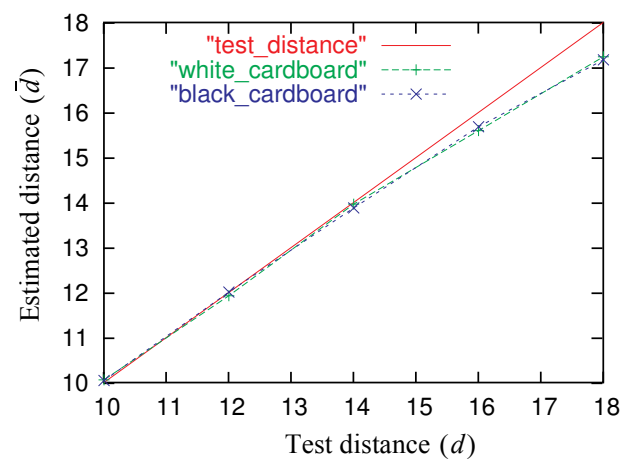

Fig. 4. Average estimated distances versus test distances for two types of material

agrees with the results reported in [4]. As a consequence, it is advisable to obtain the reflective coefficients $C_{0}$ and $C_{1}$ at distances below $14 \mathrm{~cm}(\mathrm{e} . \mathrm{g} ., 10 \mathrm{~cm})$ in order to have a reliable characterization of the measured surface.

Table 3 shows the average and standard deviation of the reflective coefficients obtained for five different materials at a test distance of $10 \mathrm{~cm}$. Due to the small standard deviations associated with those coefficients, it is possible to perform the distance measurement process in a single run (two angular scans separated by $\Delta$ ) without significant variation with respect to the average values. 
Table 3. Average Reflective Coefficients of Different Materials Estimated at the Same Test Distance $(10 \mathrm{~cm})$

\begin{tabular}{|c||c|c|c|c|}
\hline Test Material & $\overline{C_{0}}$ & $\sigma_{C_{0}}$ & $\overline{C_{1}}$ & $\sigma_{C_{1}}$ \\
\hline \hline White thin cardboard (w_card) & 16.8 & 1.39 & -7.6 & 0.97 \\
\hline Black thin cardboard (b_card) & 13.8 & 0.76 & -6.5 & 0.56 \\
\hline Unfinished brown wood (u_wood) & 14.4 & 1.42 & -3.9 & 1.10 \\
\hline Rugged gray wall (r_wall) & 11.9 & 1.3 & -5.2 & 1.15 \\
\hline Shiny brown wood (s_wood) & 7.37 & 0.38 & -2.9 & 0.41 \\
\hline
\end{tabular}

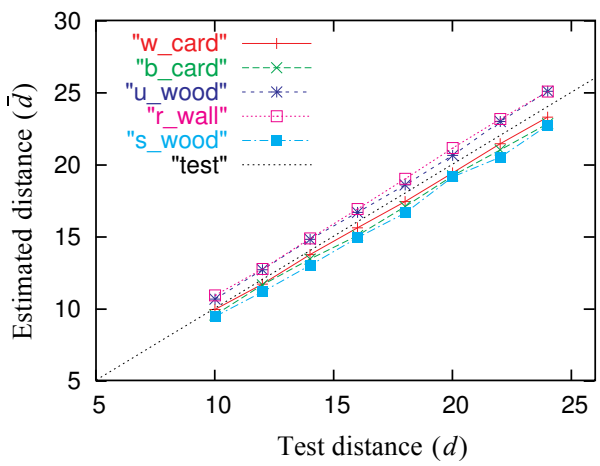

Fig. 5. Estimated distances versus test distances for five different materials by considering reflective coefficients measured at $10 \mathrm{~cm}$

Once $C_{0}$ and $C_{1}$ have been computed, (6) makes it possible to estimate the current distance $d$ between the sensor and the same surface from a single sensor reading $E_{0}^{d}$ obtained with the sensor perpendicular to the surface. This condition can be guaranteed at every distance by making a small angular scan that allows to determine the maximum reading. Fig. 5 shows the estimated distances obtained by applying the aforementioned procedure to the five previous materials, by considering test distances between 10 and $24 \mathrm{~cm}$.

The sensor response at distances above $24 \mathrm{~cm}$ is not accurate enough. In this work, both reflective coefficients were obtained from two angular scans taken at 10 and $12 \mathrm{~cm}$ respectively (Table 3 ). These results show that the distance error is kept within a small interval approximately between $0.8 \mathrm{~mm}$ and $1 \mathrm{~cm}$, rather independently of the measuring distance and the surface material.

The sum of reflective coefficients can also be utilized as an indication of the light reflective properties of the surface being measured and, hence, as a characterization of its material. For example, Table 4 shows the average sum of the coefficients whose averages and standard deviations have been presented in Table 3.

The Kolmogorov-Smirnov test [6] has been applied in order to determine whether the sums computed for each material are significantly different to the sums corre- 
Table 4. Average Sum of Reflective Coefficients of Different Materials Estimated at the Same Test Distance $(10 \mathrm{~cm})$

\begin{tabular}{|c|c|c|}
\hline Test Material & $\overline{C_{0}+C_{1}}$ & $\sigma_{C_{0}+C_{1}}$ \\
\hline \hline White thin cardboard (w_card) & 9.270 & 0.433 \\
\hline Black thin cardboard (b_card) & 7.307 & 0.234 \\
\hline Unfinished brown wood (u_wood) & 10.502 & 0.328 \\
\hline Rugged gray wall (r_wall) & 6.77 & 0.236 \\
\hline Shiny brown wood (s_wood) & 4.420 & 0.095 \\
\hline
\end{tabular}

sponding to every other material and, hence, it is possible to distinguish among the various materials. The K-S test applied to the samples belonging to every pair of tested materials returns a significance level that indicates whether the two samples are drawn from either the same or different probability distributions.

In particular, small significance levels indicate that the datasets belong to different distributions. In our case, the significance level obtained for every pair of materials is below 0.10 (significance levels below 0.15 imply different distributions, while those close to one indicate a same distribution). Hence, there is significant difference among the five materials that have been tested. This implies that the sum of reflective coefficients is a good characterization of the surface's material.

\section{Conclusions}

A new technique for computing the distance to a surface and characterizing its material exclusively through infrared sensors without a priori knowledge about the materials that can be encountered has been presented. Further work will determine the shape of the surfaces that are measured by analyzing the shape of the acquired angular scans, such as in [1]. We will also consider how redundancy provided by integrating adjacent sensors can help reduce the measurement errors present when a single sensor is utilized. Finally, more sophisticated models of light reflection [3] will be studied.

\section{References}

1. Aytaç, T., Barshan, B.: Differentiation and Localization of Target Primitives Using Infrared Sensors. IEEE/RSJ Int. Conf. on Intelligent Robots and Systems (2002) 105-110

2. Benet, G., Blanes, F., Simo, J.E., Perez, P.: Using Infrared Sensors for Distance Measurement in Mobile Robots. Robotics and Autonomous Systems 40 (2002) 255-266

3. Blinn, J.F.: Models of Light Reflection for Computer Synthesized Pictures, SIGGRAPH'77, Vol.11, No.2, July (1977) 192-198

4. Novotny; P.M., Ferrier, N.J.: Using Infrared Sensors and the Phong Illumination Model to Measure Distances. IEEE Int. Conf. on Robotics and Automation (1999) 1644-1649

5. Phong, B.T.: Illumination for Computer Generated Pictures. Communications of the ACM, 18(6), June (1975) 311-317

6. Press, W.H., Teukolsky, S.A., Vetterling, W.T., Flannery, B.P.: Numerical Recipes in C++: The Art of Scientific Computing, Cambridge University Press, 2nd Ed. (2002) 